\title{
Design of an Intelligent Tutorial System based on Ontologies
}

\author{
Abderrahim El Mhouti \\ Laboratoire de l'Informatique, \\ Recherche Opérationnelle et \\ Statistique Appliquée, \\ Abdelmalek Essaadi University, \\ Faculty of Science, \\ Tetouan, Morocco \\ B.P. 7, M'DIQ, Morocco
}

\author{
Azeddine Nasseh \\ Laboratoire de l'Informatique, \\ Recherche Opérationnelle et \\ Statistique Appliquée, \\ Abdelmalek Essaadi University, \\ Ecole Normale Supérieure, \\ B.P. 209, Martil, Morocco
}

\author{
Mohamed Erradi \\ Laboratoire de l'Informatique, \\ Recherche Opérationnelle et \\ Statistique Appliquée, \\ Abdelmalek Essaadi University, \\ Ecole Normale Supérieure, \\ B.P. 209, Martil, Morocco
}

\begin{abstract}
Today, environments of e-learning aim increasingly to give the learner an active role in learning in order to build their knowledge. They allow thereby integrating a more learnercentered vision. However, learners show differences between learning modes that suits them best. The problem posed is how to propose to the learner a teaching sequence adequate to his/her profile.

This work is at the heart of this issue. It is interested in contributing on ontologies and cognitive theories to describe the learning preferences within the Intelligent Tutorial Systems (ITS). The objective of this paper is to propose a model of ITS, based on ontologies and cognitive theories, for adapting the learning resources proposed to a learner according to his/her learning preferences. The determination of these preferences is done through the analysis of learner behavior relying on his/her indicator MBTI (Myers Briggs Type Indicator). The proposed model integrates the main functionalities of an ITS: profiling, updating of the profile, selection, adaptation and presentation of adequate resources.
\end{abstract}

\section{General Terms}

Technology, e-learning, adaptation, ontologies.

\section{Keywords}

Learner, learning style, profiling, adaptation model, ontology, MBTI, Intelligent Tutorial Systems.

\section{INTRODUCTION}

Nowadays, with the advent of the Internet, new Information Technology and Communication (ICT) profoundly improve our ways of getting informed, communicating and training us. This technological emergence brings up a new mode of learning known as e-learning.

However, learning with digital online environments takes place in a very different context of traditional learning, in which human interactions become mediated. In this new environment, the problem posed is how to propose the learner, who finds himself alone in front of the machine, an learning experience which is appropriate to his/her learning preferences? In this context, the contribution of ontologies in the e-learning to increase intelligence in Intelligent Tutorial Systems (ITS) as well as in the design of these environments has been amply demonstrated.

This article highlights the contributions of ontologies and cognitive theories in the description of learning preferences in an ITS. The aim of this work is to propose a model of ITS to adapt the resources and services offered to a learner according to their learning preferences. The model allows to refine and complete the description of learning style preferred by the learner using the profiling process, based on the theory of psychological types of MBTI (Myers-Briggs Type Indicator).

In this paper, the approach adopted consists to present the scientific context and the objective of this study. Then, the paper focuses on the concept of "learning style" and exposes the contributions of ontologies in educational systems, more specifically in the profiling process. In the next section, this work presents the MBTI tool for describing learning styles. Then, the paper describes the different components of the proposed model of ITS and presents its architecture. Finally, the paper explains the functionalities of the model and concludes with perspectives of this research.

\section{SCIENTIFIC CONTEXT AND OBJECTIVE}

To be able to place the learner at the center of the learning process, e-learning environments need to adapt to the needs of the learner according to his/her behavior. Several research studies have proposed models relating to various aspects of a person (personality, behavior, preferences, etc...) for adapting the learning content to the learner profile. In this context, there are several initiatives to use ontologies in e-learning. These ontologies play a key role in holding as an amplifier of intelligence and sharing and reuse of knowledge. This thanks to the mediation made by the computer for the dissemination of knowledge among the various actors [1].

The objective of the work presented in this paper is to study how it is possible to integrate models resulting from cognitive theories and ontologies in the e-learning applications. The cognitive theories allow describing profiles of learners and in particular their learning preferences and the ontologies allow to annotate educational resources and to implement the resulting models at the computers. The goal is to propose a model of ITS to adapt the resources and services offered to a learner according to his learning preferences.

\section{LEARNING STYLE}

The learning style is an important factor to take into account in an ITS. It is defined as the set of mental processes used by the individual to perceive and process optimally information. Its personalization then involves the estimation of differences in learning styles, approaches to learning and levels of intellectual development of students [2]. The concept of 
learning style can be used in ITS to present information based on individual characteristics of learners [3] such as different objectives, preferences and knowledge [4] maintained in a multidimensional learner model [5].

\section{ONTOLOGIES AND EDUCATION SYSTEMS}

\subsection{Contributions of ontologies in e- learning}

In the domain of e-learning, the contribution of ontologies in the design of ITS has been amply demonstrated [6]. These ontologies were the basis of navigation offering forms of acquisition other than those proposed in the course of classical learning which are generally linear type [7]. They are also used as part of an educational approach based on collaborative writing for the structuring of hyperbooks and making inferences [8].

In an ITS, the use of ontologies is quite appropriate because it allows better interoperability and data exchange [9]. Ontologies are also used for searching and indexing various educational resources [10].

\subsection{Ontologies and "profiling"}

In the context of ITS, ontologies represent a solution to describe the learning preferences of the learners: 'profiling'.

Profiling is the activity which consists on analyzing the behavior of a learner in order to deduce his profile. The profile is considered as a set of data which modelize certain characteristics of the learner [11].

In an ITS, the activity of profiling consists on defining the values of the manipulated elements by the various models, such as: the knowledge model and the student model. In this context, several studies have developed models for the representation of knowledge of learners. Some authors advocate the use of different ontologies for each of these models [12], [13], [14], [15].

This work proposes an approach based on ontologies for describing learner profiles, and more specifically for the representation of styles (preferences) of learning of a learner in an ITS. To do this, the proposed approach uses the tool MTBI (Myers Briggs Type Indicator) to describe these learning preferences.

\section{THE MBTI TOOL FOR THE DESCRIPTION OF LEARNING PREFERENCES}

The MBTI (Myers Briggs Type Indicator) is a model of psychological assessment determining the psychological type of a subject, following a method proposed in 1962 by Isabel Briggs Myers and Katherine Cook Briggs. It serves as a tool in the identification of psychological dominant of persons in contexts related to management or to the problems in the context of interpersonal relationships. This model is based on the theory of psychological types of MBTI and directly inspired the theory of psychological types of Carl Gustav Jung [16].

The MBTI describes with finesse the functioning of the person highlighting preferences spontaneous concerning four dimensions. Each dimension has two opposite poles. Figure 1 shows the four dimensions of the MBTI personality.

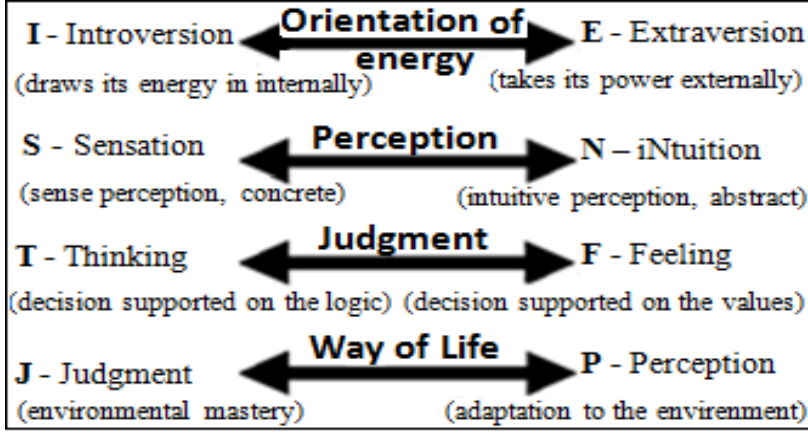

Fig 1: The four dimensions of the MBTI personality

The characteristics of each MBTI model preference are described in Table 1.

Table 1. Main characteristics of each MBTI preference

\begin{tabular}{|c|c|}
\hline rsion & Introversion \\
\hline $\begin{array}{l}\text { Prefers brining energy } \\
\text { outside oneself, at others. }\end{array}$ & $\begin{array}{l}\text { Prefers getting energy from } \\
\text { the inside world of ideas, } \\
\text { emotions, feelings. }\end{array}$ \\
\hline $\begin{array}{l}\text { Sensing } \\
\text { Prefers concentrating on the } \\
\text { information obtained from } \\
\text { the five senses and the } \\
\text { practical applications. }\end{array}$ & $\begin{array}{l}\text { Intuition } \\
\text { Prefers concentrating on the } \\
\text { structure, the rapports and the } \\
\text { possible meanings. }\end{array}$ \\
\hline $\begin{array}{l}\text { Thinking } \\
\text { Prefers making decisions } \\
\text { based on logic and en the } \\
\text { objective analysis of causes } \\
\text { and effects. }\end{array}$ & $\begin{array}{l}\text { Feeling } \\
\text { Prefers making decisions } \\
\text { based on appreciation } \\
\text { progress, and taking into } \\
\text { consideration what is } \\
\text { important for people. }\end{array}$ \\
\hline $\begin{array}{l}\text { Judging } \\
\text { Appreciates a planned and } \\
\text { organized approach to life. It } \\
\text { prefers decisions- making. }\end{array}$ & $\begin{array}{l}\text { Perceiving } \\
\text { Appreciates a flexible and } \\
\text { spontaneous approach, and } \\
\text { prefers keeping options. }\end{array}$ \\
\hline
\end{tabular}

Combining poles of different dimensions (E-I, S-N, T-F, J-P), the MBTI indicator allows to distinguish sixteen psychological types (Table 2).

Table 2. The 16 psychological types of MBTI

\begin{tabular}{|c|c|c|c|}
\hline INTP & INFP & ISTP & ISFP \\
\hline INTJ & ENFP & ISTJ & ISFJ \\
\hline ENTJ & INFJ & ESTP & ESFP \\
\hline ENTP & ENFJ & ESTJ & ESFJ \\
\hline
\end{tabular}

According to the theory of psychological types, each has a natural preference for one of opposite poles of each of the four dimensions. When someone uses his preferred pole, it generally succeeds better and it feels more competent, natural and dynamic. Generally speaking, when they tackle new or difficult topics, students learn easily when they have the opportunity to use their preferred learning style. 


\section{A MODEL OF ITS BASED ON ONTOLOGIES}

The proposed model of ITS is implemented using ontologies. The process of profiling rests on MBTI indicator of learners.

\subsection{Ontologies implemented}

The proposed model of ITS has four main ontologies: Learner, Resource, Domain, and Strategy of teaching/learning. Each ontology is composed of several subontologies (simplified ontologies). The four ontologies are interconnected and can describe the two main models of the adaptive teaching /learning : Pedagogical model and Domain of knowledge model.

\subsubsection{Learner ontology}

The "Learner" model is defined as an ontology $\mathrm{O}_{\text {Learner }}$ which comprises various characteristics of a user. This work proposes to describe a student in four facets. These facets are described as sub-ontologies (SO) : $\mathbf{O}_{\text {Learner }}=\left\{\mathbf{S O}_{\text {Identity }}\right.$, $\left.\mathrm{SO}_{\text {Preferences }}, \mathrm{SO}_{\text {Capacity }}, \mathrm{SO}_{\text {History }}\right\}$.

The sub-ontology "Identity" $\mathbf{S O}_{\text {Identity }}$ is responsible for representing the information about a particular user. It is composed of essential and common to all users predefined attributes: name, username, language, media type, etc.

The sub-ontology "Preferences" $\mathbf{S} \mathbf{O}_{\text {Preferences }}$ is responsible for representing the preferences of learning among learners. This component is based on the theory of psychological types of MBTI. It describes the preferences of the learner as a vector of preferences. The latter is described as a conceptual vector $\mathrm{V}_{\text {Preferences }}=\{\mathrm{Ep}, \mathrm{Ip}, \mathrm{Sp}, \mathrm{Np}, \mathrm{Tp}, \mathrm{Fp}, \mathrm{Jp}, \mathrm{Pp}\}$. This vector is used to specify the MBTI psychological style of the learner and thus inform about these learning preferences with a questionnaire. For example, the psychological type of a learner $\mathrm{A}_{1}$ are described as follows: $\left\{\mathrm{A}_{1},\langle\mathrm{Ep}: 18 \%\rangle\right.$, $\langle\mathrm{Ip}$ :

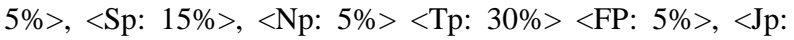
$20 \%\rangle,\langle\mathrm{Pp}: 2 \%\rangle$ \}, which indicates that this learner has the psychological type (profile) ESTJ.

The sub-ontology "Capacity" (knowledge) SO $_{\text {Capacity }}$ is responsible for representing or giving a knowledge level of a learner for a concept. This knowledge is modeled by a stereotype (class of individuals) can be obtained with a level test (MCQs). Possible values are "very low", "low", "medium", "good" and "excellent". For example, levels of learner $\mathrm{A}_{1}$ are described as follows: $\left\{\mathrm{A}_{1},<"\right.$ Network", "definition", "Medium"> <"Network", "description", "low"> <"OSI", "example", "Medium">\}.

The sub-ontology "History", $\mathbf{S O}_{\text {History }}$ is responsible for keeping track of the status of the historic of a learner (memorizing the navigation and reading resources documents). This representation allows giving the date of the consulting of a resource or also the paths of route for the navigation order. For example, the path of the learner $A_{1}$ is : $\left\{\mathrm{A}_{1}, \quad<" N e t w o r k "\right.$, "definition", "01/06/13">, <"Network", "description", "10/10/13" >\}.

\subsubsection{Resource ontology}

The "Resource" model is defined as a $\mathrm{O}_{\text {Resource }}$ ontology including various characteristics of an educational resource. This work proposes to describe a digital resource on two facets. These facets are described as sub-ontologies : $\mathrm{O}_{\text {Resource }}$ $=\left\{\mathrm{SO}_{\text {Description }}, \mathrm{SO}_{\text {Catgory }}\right\}$.
The first, $\mathbf{S O}_{\text {Description, }}$ describes the educational characteristics of the resource (author, title, language, media, time, ...) and is modeled by a set of pairs "attribute-value". This part is similar to the metadata described in existing standards such as LOM. For example, the resource $R_{1}$ is described by the following educational characteristics: $R_{1}=\{$ langage, "french">, $<$ media, "text", "video", "image">< <author, "El Mhouti"> \}.

The second, $\mathbf{S O}_{\text {Catgory }}$, used to classify learning resources into different categories based on their content, their uses and purposes of such uses. Each category is described by a concept which consists of the following elements: a title, a descriptive, a vector of use. The latter is described as a conceptual vector $\mathrm{V}_{\mathrm{Use}}=\{\mathrm{Eu}, \mathrm{Iu}, \mathrm{Su}, \mathrm{Nu}, \mathrm{Tu}, \mathrm{Fu}, \mathrm{Ju}, \mathrm{Pu}\}$.

The conceptual vector of a learning resource allows specifying that his content is more suited (adequate) for a given learning style, so for a MBTI psychological style. For example, an resource $R_{1}$ with the vector $V_{U s e}=\left\{R_{1}\right.$, $<E u$ $: 5 \%\rangle,\langle\mathrm{Iu}: 20 \%\rangle,\langle\mathrm{SU}: 30 \%\rangle\langle\mathrm{Nu}: 10 \%\rangle\langle\mathrm{Tu}: 15 \%\rangle,\langle\mathrm{Fu}:$ $5 \%\rangle,\langle\mathrm{Ju}: 14 \%\rangle,\langle\mathrm{Pu}: 1 \%\rangle\}$ indicates that this resource is more suited to a ISTJ profile knowing how to conduct a reflection (Iu 20\%), which instead focuses on practical aspects (Su: $30 \%)$, (Tu: $15 \%$ ) that is motivated by the realization, etc. The allocation of settings of a resource is made by the designer (or annotator) knowing the content, purpose and possible use of this resource. This assignment is carried via an ergonomic interface (forms, questionnaires, etc.). Masking the technical details when the testimony of a resource. Thereafter, the value of a vector of the use of a resource can be changed (adjusted) manually by the designer of the resource or automatically by the system based on traces of future uses of this resource by different learner profiles.

\subsubsection{Domain ontology}

The "Domain" ontology, $\boldsymbol{O}_{\text {Domain }}=\left\{\boldsymbol{S O}_{\text {Computer science, }}\right.$, $\boldsymbol{S} \boldsymbol{O}_{\text {Mathematics, }} \boldsymbol{S} \boldsymbol{O}_{\text {Physics, }}$ etc. $\}$, describes the concepts of a domain of knowledge that correspond to an educational domain (mathematics, computer science, chemistry, law, etc.). We can have several domains ontologies, each being specific to a teaching discipline and she describes the different concepts. A concept is a unique notion of the domain of knowledge represented by a name. For example, the ontology of the domain of computer science includes the following concepts: database, programming languages, computer networks, etc.

\subsubsection{Pedagogical Strategies ontology}

The pedagogical strategy model is defined as an ontology, $\mathrm{O}_{\text {Strategies, }}$ comprising all the models of teaching/learning (transmissive, constructivist, socioconstructivist, etc..), the methods and approaches, which will determine the choice of techniques, materials and educational situations, relative to the object and the goal of learning. Teaching strategies can be chosen according to the learning style of the learner.

This work proposes to decompose the ontology "Strategy" into two sub-ontologies (SO), according to the Lasnier decomposition [17] : sub-ontology "Teaching strategies" and sub-ontology "Learning strategies": $\mathrm{O}_{\text {Strategies }}=\left\{\mathrm{SO}_{\text {Teaching }}\right.$, $\left.\mathrm{SO}_{\text {Learning }}\right\}$.

The sub-ontology "Teaching strategies", $\mathrm{SO}_{\text {Teaching, includes }}$ the masterful strategies (presentation, practical demonstration), individual work strategies (independent practice, individual work session, problem-based learning), interactive strategies (group discussion, role play, modelling, guided practice, learning by steps) and constructivist and 
socio-constructivist strategies (peer tutoring, mentoring, teamwork, cooperative learning, project-based learning, case studies).

The sub-ontology "Learning strategies", $\mathrm{SO}_{\text {Learning }}$, includes cognitive strategies (activation, acquisition, development, organization of knowledge, integration, transferring), affective strategies (reception, motivation, stress management, cooperation, conflict resolution) management strategies (time, material resources, human resources, environment) and metacognitive strategies (planning, monitoring, controlling and evaluating).

\subsection{Modules implemented}

The used ontologies generate two extensional modules (Figure 2): "Learner" module and "Resource" module.

\subsubsection{Learner module: $M_{\text {Learner }}$}

This module is composed of three sub-modules (SM): $\mathrm{M}_{\text {Learner }}$ $=\left\{\mathbf{S M}_{\text {Preferences}}, \mathbf{S M}_{\text {Identity }}, \mathbf{S M}_{\text {Acquis }}\right\}$. The three sub-modules are the result of an instantiation, respectively, of sub- ontologies, $\mathbf{S} \mathbf{O}_{\text {Preferences}}$ SO $\mathbf{S O}_{\text {Identity }}, \mathbf{S O}_{\text {Capacity. The }} \mathbf{S M}_{\text {Acquis }}$ used to describe knowledge acquired through learning by specifying the level of mastery of various concepts of domain ontology. For example, a level of "good" to " Relational databases".

\subsubsection{Resource module: $M_{\text {Resource }}$}

This module is composed of two sub-modules: $\mathbf{M}_{\text {Resource }}=$ $\left\{\mathbf{S M}_{\text {Description }}, \mathbf{S M}_{\text {Category }}\right\}$. The $\mathbf{S M}_{\text {Description }}$ component is the result of an instantiation of the sub-ontology $\mathbf{S O}_{\text {Description, }}$ enriched with annotations by concepts coming from the ontology $\mathrm{O}_{\text {Domain }}$. These annotations are used to describe the semantics associated to the content of the resource. The $\mathbf{S M}_{\text {Category }}$ is a component used to describe the semantics associated with pedagogical uses associated with the resource.

The following Fig. 2 shows the architecture of the proposed model of ITS, that allows the adaptation of learning resources to learners' profiles.

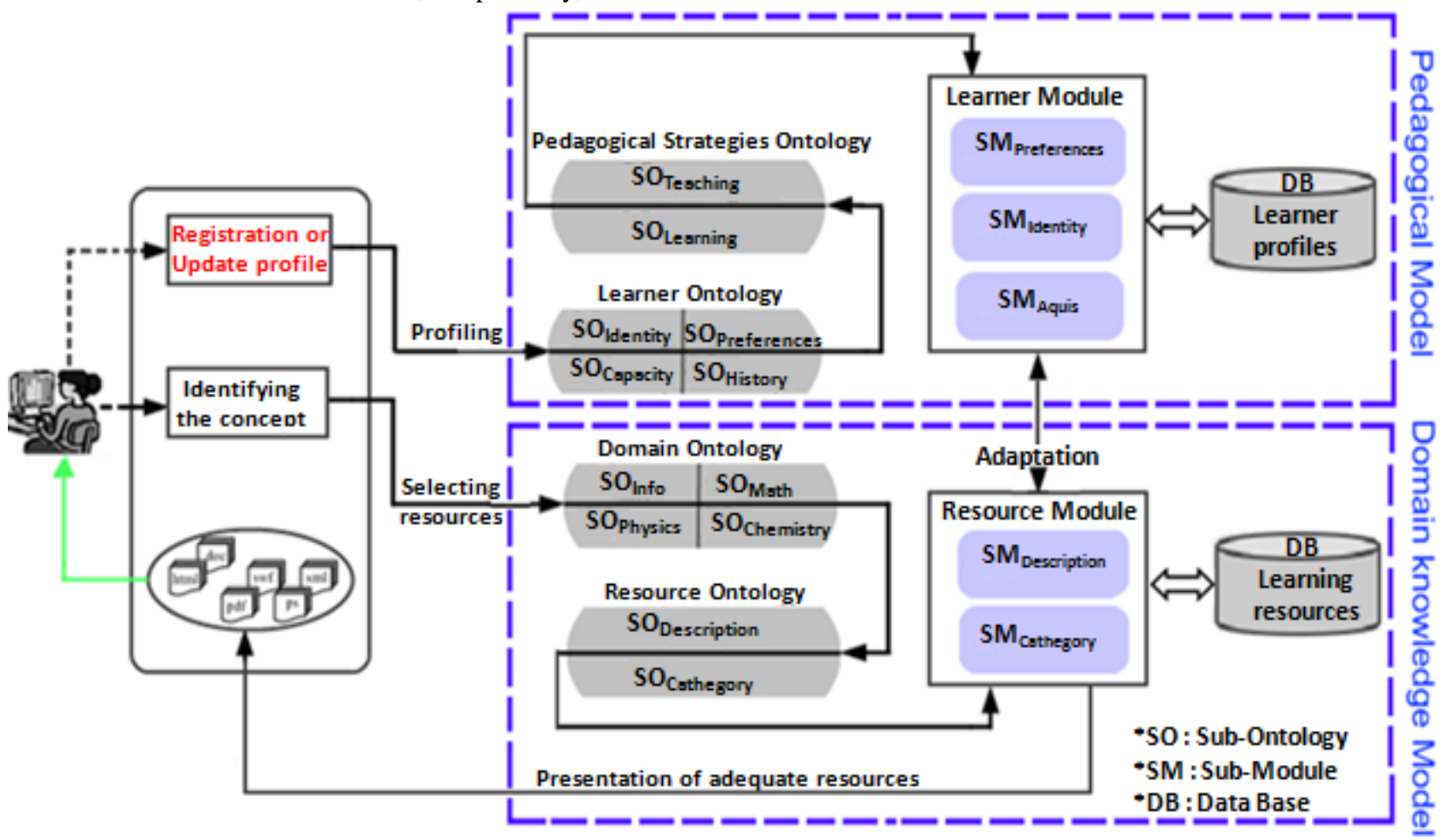

Fig 2: Architecture of the proposed model of ITS

\section{FUNCTIONING OF THE PROPOSED MODEL}

In the proposed model, the process of generating of pedagogical resources, adapted to the learner profile, is performed in several steps :

\subsection{Profiling}

When registering a new learner, the system proposes him/her a questionnaire to determine his/her psychological type. After completing and validating the questionnaire, the system calculates and stores the result in a database of profiles of learners.

\subsection{Update of profile}

A learner already registered can update him/her profile by changing the values representing the level of knowledge and this for a number of resources of a given concept. The historic, modeled with the sub-ontology $\mathbf{S O}_{\text {History }}$, will also evolve dynamically and automatically as and when the learner will follow new knowledge.

\subsection{Resources selection}

The learner connects to the system via a user-friendly interface allowing him to describe his request (search for resources, etc.). This request is described using concepts from the domain ontology $\left(\mathrm{O}_{\text {Domain }}\right)$. The system is responsible for a 
pairing between the semantic of request and the semantic of contents stored in the database of resources and annotated by concepts coming from the ontology $\mathrm{O}_{\text {Domain }}$. This pairing is accomplished by using a distance $\mathrm{D}_{\text {Content }}$ which compares the query structure with annotations of those resources.

\subsection{Adaptation}

The result of the previous step is a list of content $\mathrm{L}_{\text {Content }}$. The system compares the semantics of resource uses from the list with the description of the learning preferences of the student who submitted the initial request. This comparison is performed using a distance $\mathrm{D}_{\text {use }}$ which calculates the distance (in the vectorial sense) between the vector $V_{\text {Preferences, }}$ describing learning preferences of the learner, and the vector $\mathrm{V}_{\mathrm{Use}}$ of usage of each resource.

\subsection{Presentation of adapted resources}

At the end of the previous two steps (selection and adaptation), the system has a set of educational resources which all correspond physically and semantically to learner needs. A list $\mathrm{L}_{\mathrm{Use}}$ of adequate resources is then proposed to the learner. The latter made his choice among the items on this list as he/she may reject the entire list. The decision of the learner is stored in the system. Thus, further processing in the footsteps of course of the learner can be exploited to refine the profile of learner or the descriptions of resources usages.

\section{CONCLUSION AND PERSPECTIVES}

Since the birth of Web 2.0, learning systems attempt to increase the efficiency, quality and relevance associated with their models. In this context, different types of ontologies (domain, user, behavior, etc.) have been proposed.

This article presents a model of ITS, based on ontologies and cognitive theories, to adapt educational resources to learners' profiles, especially their learning preferences.

The proposed model is based on the theory of psychological types of MBTI. It revolves around four main ontologies: Learner, Resource, Domain, and Strategies of teaching/ learning. To lead the student to achieve the learning objective, these ontologies are used to select the appropriate content and adopt a suitable strategy of teaching/learning.

As part of the research actions initiated in the Laboratory of Computer Science Operational Research and Applied Statistics, further research is underway to implement and integrate the model in a platform of e-learning in the form of complementary modules.

\section{REFERENCES}

[1] Mizoguchi, R. (2004). Le rôle de l'ingénierie ontologique dans le domaine des EIAH, Revue Sticef, vol. 11.

[2] Felder, R. M. and Brent R. (2005). Understanding Student Differences, Journal of Engineering Education, vol. 94(1), (pp. 57-7).

[3] Barker T.,Jones S., Britton C. and Messer D. (2002). The Use of a Co-operative Student Model of Learner
Characteristics to Configure a Multimedia Application, User Modeling and User-Adapted Interaction, vol. 12( 2 -3), (pp. 207-241).

[4] Brusilovsky P. (2001). Adaptive Hypermedia, User Modeling and User-Adapted Interaction.

[5] Zukerman I. and Litman D. (2001). Natural Language Processing and User Modeling: Synergies and Limitations, User Modeling and User-Adapted Interaction, vol. 11(1-2), (pp. $129-158)$.

[6] Bermudez L., Piasecki M.,. (2006). Metadata Community Profiles for the Semantic Web. Geoinformatica, Springer, (10) pp.159-176.

[7] Sander E., Meunier J. and Boscminé C. (2004). Approche ontologique et navigation dans un EIAH. Revue Sticef, vol. 11.

[8] Falquet G., Nerima L. and Ziswiler J. (2004). Utilisation des ontologies dans les environnements d'écriture collaborative d'hyperlivres, expériences et perspectives. Revue Sticef, vol. 11.

[9] Cristea A. I. (2004). What can the Semantic Web do for Adaptive Educational Hypermedia? Educational Technology \& Society, vol. 7 (4), (pp. 40-58).

[10] Azouaou F. (2006). Modèles et outils d'annotations pour une mémoire personnelle de l'enseignant, Thèse de Doctorat, spécialité Informatique, Université Joseph Fournier, Grenoble, France.

[11] Piombo C. (2007). Modélisation probabiliste du style d'apprentissage et application à l'adaptation de contenus pédagogiques indexés par une ontologie, Thèse de doctorat, IRIT, France.

[12] Sosnovsky S. (2006). Integration of Two Domain Conceptualizations in a Single Adaptive Hypermedia System, Workshop on Applications of Semantic Web Technologies for e-Learning (SW-EL@AH'06), Ireland.

[13] B. Castello, W. Neto, O. Alvaro et F. Gauthier, Sharing and Reusing Information on Web-Based Learning, SWEL@AH’06, Dublin, Ireland, June 21-23 2006.

[14] Morales R., Van Labeke N. and Paul B. (2006).Towards a Learner Modelling Engine for the Semantic Web, Workshop on Applications of Semantic Web Technologies for e-Learning (SW-EL@AH’06), Ireland.

[15] A. Ounnas, I. Liccardi, H. Davis., D. Millard et S. White, Towards a Semantic Modeling of Learners for Social Networks, SW-EL@AH’06, Dublin, Ireland, June 21-23 2006.

[16] Todd C.R. (2004). Myers-Briggs Type Indicator®, The Skeptic's Dictionary.

[17] Lasnier F. (2000). Réussir la formation par compétences. Montréal : Guérin. 